\title{
Incidence and risk factors for surgical site infection following elective foot and ankle surgery: a retrospective study
}

\author{
Jinghong Meng ${ }^{1 \dagger}$, Yanbin Zhu ${ }^{2 \dagger}$, Yansen $\mathrm{Li}^{3}$, Tao Sun ${ }^{4}$, Fengqi Zhang ${ }^{3}$, Shiji Qin ${ }^{3}$ and Haitao Zhao ${ }^{3^{*}}$
}

\begin{abstract}
Background: This study aimed to investigate the incidence of surgical site infection (SSI) in elective foot and ankle surgeries and identify the associated risk factors.

Methods: This was designed as a retrospective study, including patients who underwent elective surgery of foot and ankle between July 2015 and June 2018. Data on demographics, comorbidities, and perioperative parameters were collected from the medical records, the laboratory report, the operation report, and the outpatient follow-up registration database. SSI was defined in accordance with the Center for Disease Control criteria. Univariate and multivariate logistic regression analyses were used to identify the independent risk factors for SSI.

Results: A total of 1201 patients undergoing 1259 elective foot/ankle surgeries were included, of whom 26 (2.1\%) had an SSI, representing an incidence rate of $1.3 \%$ for superficial SSI and $0.8 \%$ for deep SSI, respectively. The results for organism culture showed Pseudomonas aeruginosa in 7 cases, methicillin-resistant Staphylococcus aureus (MRSA) in 6, methicillin-susceptible Staphylococcus aureus (MSSA) in 5, methicillin-resistant coagulase-negative Staphylococci (MRCNS) in 2, Escherichia coli in 2, and Proteus mirabilis in 1 case. Five factors were identified to be independently associated with SSI, including prolonged preoperative stay $(\mathrm{OR}, 1.21 ; 95 \% \mathrm{Cl}, 1.09$ to 1.30), allograft or bone substitute (OR, 3.76; 95\% Cl, 1.51 to 5.30), elevated FBG level (OR, 1.17; 95\% Cl, 1.04 to 1.26), lower ALB level (OR, 2.33; $95 \% \mathrm{Cl}, 1.19$ to 3.05$)$, and abnormal NEUT count (OR, $1.72 ; 95 \% \mathrm{Cl}, 1.27$ to 2.12$)$.

Conclusions: SSI following elective foot and ankle surgeries is low, but relatively high in forefoot surgeries, requiring particular attention in clinical practice. Although most not modifiable, these identified factors aid in risk assessment of SSI and accordingly stratifying patients and therefore should be kept in mind.
\end{abstract}

Keywords: Surgical site infection, Foot and ankle surgery, Multivariate analysis, Risk factors

\footnotetext{
* Correspondence: drzhaohaitao@126.com

†inghong Meng and Yanbin Zhu contributed equally to this work. ${ }^{3}$ Department of Foot and Ankle Surgery, The 3rd Hospital of Hebei Medical University, No. 139 Ziqiang Road, Shijiazhuang 050051, Hebei, People's Republic of China

Full list of author information is available at the end of the article
}

(c) The Author(s). 2020 Open Access This article is licensed under a Creative Commons Attribution 4.0 International License, which permits use, sharing, adaptation, distribution and reproduction in any medium or format, as long as you give appropriate credit to the original author(s) and the source, provide a link to the Creative Commons licence, and indicate if changes were made. The images or other third party material in this article are included in the article's Creative Commons licence, unless indicated otherwise in a credit line to the material. If material is not included in the article's Creative Commons licence and your intended use is not permitted by statutory regulation or exceeds the permitted use, you will need to obtain permission directly from the copyright holder. To view a copy of this licence, visit http://creativecommons.org/licenses/by/4.0/ The Creative Commons Public Domain Dedication waiver (http://creativecommons.org/publicdomain/zero/1.0/) applies to the data made available in this article, unless otherwise stated in a credit line to the data. 


\section{Background}

Surgical site infection (SSI) represents 31\% of all hospitalacquired illness and is the most common nosocomial infection [1]. Specified in the elective orthopedic surgery, SSI was infrequent, with an incidence rate of 0.4 to $3.6 \%$ $[2,3]$. But they can lead to serious consequence, such as bone union-related issues, joint dysfunction, amputation, and even increased mortality [4]. Besides, the substantial economic and social impact of infection-related complications should also be a concern. It was documented that, irrespective of surgery type, an infection could incur additional cost of $£ 814$ and $£ 6626$ due to prolonged hospitalization, wound care, or additional surgical debridement $[5,6]$. Additionally, an infection was a major cause for readmission, accounting for $34.3 \%$ of the adverse affairs related to surgery [7].

Compared to other elective orthopedic surgeries (spinal procedure, total hip or knee arthroplasty), foot and ankle surgery had a relatively higher incidence rate of SSI, ranging from 0.5 to $6.5 \%$ [8]. Identification of risk factors associated with SSI could provide necessary information to appropriately counsel patients and aid surgeons to select targeted preventive measures. In the study by Ralte et al. [9], authors found the strict infection policy control policies that focused on surgical and environmental risk factors could reduce $50 \%$ of SSIs in elective foot and ankle surgery. By far, however, epidemiologic data on SSI following elective foot and ankle surgeries remain scarce. As far as we know, only very few studies focused on this subject and identified some risk factors, including alcohol use [10], preoperative narcotic use [10], advanced age [11], prolonged tourniquet time [11], tobacco use [11], complicated diabetes [4], perioperative antibiotics, and surgeon experience [12]. However, most of these risk factors were in controversy or inconclusive, which might be compromised by the small sample size or only inclusion of a specific population (diabetes mellitus, elderly, or foot and ankle trauma). It was possible that these variable results from studies might not be applicable to a general population undergoing elective foot and ankle surgeries.

Given that, we designed this study, with purposes to determine the incidence rate of SSI in patients undergoing clean elective foot and ankle surgery and secondly to identify the independent risk factors associated with SSIs.

\section{Materials and methods}

\section{Study design, inclusion and exclusion criteria}

This was a retrospective study and designed in accordance with the principles outlined in the Declaration of Helsinki. It had been approved by the institutional review board of the 3rd Hospital of Hebei Medical University. Between July 2015 and June 2018, all patients aged 18 years or older who underwent clean elective foot and ankle surgery were confirmed by querying electronic medical records (EMR). The exclusion criteria were as follows: foot and ankle trauma, revision surgery, isolated ankle arthroscopy, hardware removal, previous foot/ ankle surgery, foot/ankle ulcerations, patients with incomplete EMR data, death within the hospitalized stay, or follow-up period $<12$ months.

According to our policy, patients who are to be operated are compulsory to soak their feet in the simple soap solution the night before the operation, but are no need of hair removal. Then, before surgery, the surgical site is cleansed with an antiseptic soap solution and broad disinfection with iodine solution is performed by three times. Generally, single dose of prophylactic antibiotic (cefuroxime, ceftriaxone, or ceftazidime) is administered intravenously $30 \mathrm{~min}$ before the surgery, and in some cases exceeding $3 \mathrm{~h}$, a second dose is given.

\section{Definition of SSI and confirmation of cases}

SSI is confirmed by the documented signs or symptoms of infection in the EMR and bacteria culture results or drug sensitivity available in the microbial culture report. SSI is diagnosed based on the criteria proposed by the Center for Disease Control (CDC) [13], wherein SSI is an incisional infection that occurs within 30 days after surgery if no implant is left in place or within 1 year if an implant is left in place. SSI is classified as deep and superficial SSI. The deep SSI involves deep soft tissue and meets at least one of the following: persistent wound discharge or dehiscence; visible abscess or gangrenosis that requires surgical debridement, implant exchange, or removal; and an infection that involves only the skin or subcutaneous tissue, presenting wound problem (redness, swelling, hot, pain) but does not meet the diagnosis criteria of deep SSI is deemed to be a superficial SSI.

We confirm the SSI cases if we found they were (1) documented by the treating surgeon in the inpatient medical record, (2) documented in the outpatient follow-up registration database, and (3) confirmed by the telephone visit by the end of 1 year postoperatively. It is of note that SSI cases which were treated in our institution and in other institutions or untreated to resolve were all included in this study.

\section{Data collection}

Two researchers ( $\mathrm{Y} \mathrm{Li}$ and T Sun) inquired patients' EMR, scheduled follow-up registration database and microbial culture reports for patients' demographics, lifestyles, comorbidities, surgery-related data, and laboratory biomarkers.

The detailed data for each patient were age, sex, body mass index (BMI), tobacco use, alcohol consumption, and comorbidities (hypertension, diabetes mellitus (DM), chronic heart disease, chronic pulmonary disease, peripheral vascular disease, and rheumatoid disease). 
Due to the retrospective design, pack-year history of tobacco users or volume of alcohol consumption was not available. BMI $\left(\mathrm{kg} / \mathrm{m}^{2}\right)$ was grouped based on the criteria suitable for Chinese populations: < 18.5 (underweight), 18.5-23.9 (normal), 24.0-27.9 (overweight), and $\geq 28.0$ (obesity) [14, 15]. Comorbidities were confirmed mainly based on the patients' self-reported disease history at their admission. Also, the repeated measurements of blood pressure, fasting blood glucose (FBG), glucose tolerance tests, or glycosylated hemoglobin levels were used for additional diagnosis of hypertension or DM.

Surgery-related variables included preoperative stay (between admission and operation), surgeon level (senior, vice senior, attending surgeon, or resident), American Society of Anesthesiologists classification (ASA), anesthesia type, surgical duration, use of allogeneic bone or bone substitute, volume of intraoperative blood loss, intraoperative blood transfusion, intraoperative or postoperative prophylactic use of antibiotics, and postoperative drainage.

The following laboratory biomarkers were collected: preoperative white blood cell (WBC), neutrophil (NEUT), lymphocyte (LYM), red blood cell (RBC), hemoglobin (HGB), hematocrit (HCT), total protein (TP), albumin (ALB), globulin (GLOB), A/G value, and FBG. If patients have multiple laboratory biomarkers recorded during the preoperative period, the biomarkers measured at closest date before surgery were selected.

\section{Statistical analysis}

The continuous data were expressed as mean \pm standard deviation (SD) or median (interquartile range, IQR) and were evaluated consistently by the Student $t$ test or Mann-Whitney $U$ test, when appropriate. The categorical data were expressed as number and percentages and were analyzed by chi-square or Fisher's exact test, when appropriate.

The variables that were tested to be significant at statistical level $p<0.1$ in the univariate analyses were further entered into a multivariate logistic regression model to determine their independent effect on SSI, using stepwise backward elimination method. The goodness-of-fit of the final model was evaluated by the HosmerLemeshow test, with a $p>0.05$ indicating an acceptable result, and was quantitatively evaluated by Nagelkerke's $R^{2}$, with greater value indicating a better result. SPSS 23.0 software package (SPSS Inc., Chicago, IL) was used to analyze all the data.

\section{Results}

\section{General result}

Totally, 1201 patients who underwent 1259 elective surgeries at foot/ankle were included. Of them, there were 572 males (47.6\%) and 629 females (52.4\%). The mean age was 45.0 years (SD, 16.2), with patients of 30 to 64 years in predominance $(948,79 \%)$. The most commonly performed procedure was osteotomy (497, 45.8\%), followed by arthrodesis (392, 31.1\%), soft tissue procedure (63, 5.0\%), and combined procedure (307, 18.0\%). Surgical procedure was performed at median of 2 days (IQR, 1 to 4 days) after admission, and $83.0 \%(1045 / 1259)$ were performed within 3 days after admission. The median total hospital stay was 8.0 days (IQR, 6 to 13 days).

\section{Characteristics of SSI (incidence, causative bacteria type, and SSI occurrence time)}

Twenty-six SSIs occurred in 26 patients, representing an overall incidence rate of $2.1 \%$. Of them, 16 (incidence rate, $1.3 \%$ ) were superficial and 10 (incidence rate, $0.8 \%$ ) were deep SSI. The positive results of organism culture for 10 deep and 14 superficial SSIs showed Pseudomonas aeruginosa in 7, methicillin-resistant Staphylococcus aureus (MRSA) in 6, methicillin-susceptible Staphylococcus aureus (MSSA) in 5, methicillin-resistant coagulasenegative Staphylococci (MRCNS) in 3, Escherichia coli in 2, and Proteus mirabilis in 1 case. There were no SSIs that were caused by mixed bacteria. The median time at which SSIs occurred was 5.5 days after operation, ranging from 2 to 38 days postoperatively.

Stratified by surgical location, most SSIs occurred in the forefoot $(22 / 26,84.6 \%)$ and 21 were cultured positive for causative organisms, with Pseudomonas aeruginosa in 6 cases, MRSA in 6, MSSA in 4, MRCNS in 2, Escherichia coli in 2, and Proteus mirabilis in 1 case; one SSI caused by MSSA occurred at the midfoot; 2 among 3 SSIs in the hindfoot were cultured positive, with one caused by MRCNS and the other by Pseudomonas aeruginosa.

\section{Univariate analyses}

The results of univariate analyses are presented in Table 1. There were significant differences between the SSI and non-SSI groups in terms of age $(p=0.040)$, diabetes mellitus $(p<0.001)$, preoperative stay $(p<0.001)$, intraoperative bleeding $(p=0.006)$, anesthesia type $(p<0.001)$, type of bone graft $(p=0.002)$, albumin $(p<0.001), \mathrm{A} / \mathrm{G}(p<$ $0.001), \operatorname{ALT}(p=0.019), \operatorname{AST}(p=0.011), \operatorname{ALP}(p=0.020)$, GLOB $(p=0.002)$, FBG $(p=0.042)$, WBC $(p<0.001)$, NEUT $(p<0.001)$, RBC $(p<0.001)$, HGB $(p<0.001)$, $\operatorname{MCHC}(p<0.001)$, PLT $(p<0.001)$, and PDW $(p<0.034)$. The total hospital stay was 26.2 days in patients with SSI, while 10.3 days in those without SSIs $(p<0.001)$.

\section{Multivariate analyses}

We entered significant variables together with surgical duration $(p=0.074)$ and cigarette smoking $(p=0.085)$ into the multivariate logistic regression model. After adjustment for confounding factors, five independent risk 
Table 1 Association between potential risk factors and surgical site infection (SSI) in elective foot and ankle surgery

\begin{tabular}{|c|c|c|c|}
\hline Variables & Number (\%) of infection & Number (\%) of non-infection & $p$ value \\
\hline Gender (male) & $13(50.0)$ & $576(46.7)$ & 0.740 \\
\hline Age (years) & $40.3 \pm 11.5$ & $45.3 \pm 16.4$ & 0.040 \\
\hline Living place & & & 0.311 \\
\hline Rural & $16(61.5)$ & $635(51.5)$ & \\
\hline Urban & $10(38.5)$ & $598(48.5)$ & \\
\hline BMI $\left(\mathrm{kg} / \mathrm{m}^{2}\right)$ & $24.4 \pm 5.6$ & $25.4 \pm 3.9$ & 0.280 \\
\hline $18.5-23.9$ & $8(30.8)$ & $437(35.5)$ & 0.112 \\
\hline$<18.5$ & $3(11.5)$ & $38(3.1)$ & \\
\hline $24.0-27.9$ & $7(26.9)$ & $472(38.3)$ & \\
\hline $28.0-31.9$ & $6(23.1)$ & $232(18.8)$ & \\
\hline$\geq 32.0$ & $2(7.7)$ & $54(4.4)$ & \\
\hline Diabetes mellitus & $7(26.9)$ & $96(7.8)$ & $<0.001$ \\
\hline Hypertension & $4(15.4)$ & $259(21.0)$ & 0.621 \\
\hline Chronic heart disease & $3(11.5)$ & $62(5.0)$ & 0.138 \\
\hline Allergy history & $2(7.7)$ & $132(10.7)$ & 0.622 \\
\hline Preoperative stay (days) & $5.4 \pm 3.4$ & $2.8 \pm 2.9$ & $<0.001$ \\
\hline Total hospital stay (days) & $26.2 \pm 10.4$ & $10.3 \pm 11.9$ & $<0.001$ \\
\hline Intraoperative bleeding (mL) & $45.6 \pm 83.2$ & $71.8 \pm 140.1$ & 0.006 \\
\hline Surgical duration (min) & $76.5 \pm 47.0$ & $64.8 \pm 51.4$ & 0.074 \\
\hline Cigarette smoking & $8(30.8)$ & $218(17.7)$ & 0.085 \\
\hline Alcohol consumption & $5(19.2)$ & $402(32.6)$ & 0.149 \\
\hline Surgeon level & & & 0.403 \\
\hline Attending or resident & $5(19.2)$ & $167(13.5)$ & \\
\hline Senior or vice senior & $21(80.8)$ & $1066(86.5)$ & \\
\hline Surgical location & & & 0.233 \\
\hline Forefoot & $22(84.6)$ & $869(69.0)$ & \\
\hline Midfoot & $1(3.8)$ & $97(7.7)$ & \\
\hline Hindfoot & $3(11.5)$ & $293(23.3)$ & \\
\hline Procedure & & & 0.677 \\
\hline Osteotomy & $9(34.6)$ & $488(39.6)$ & \\
\hline Arthrodesis & $7(26.9)$ & $385(31.2)$ & \\
\hline Soft tissue & $1(3.8)$ & $62(5.0)$ & \\
\hline Combined two or three above & $9(34.6)$ & $298(24.2)$ & \\
\hline Anesthesia & & & $<0.001$ \\
\hline Local & $6(23.1)$ & $585(47.4)$ & \\
\hline Spinal & $12(46.2)$ & $564(45.7)$ & \\
\hline General & $8(30.8)$ & $84(6.8)$ & \\
\hline Type of bone graft & & & 0.002 \\
\hline None & $21(80.8)$ & $1150(93.3)$ & \\
\hline Autograft & $3(11.5)$ & $68(5.5)$ & \\
\hline Allograft or bone substitute & $2(7.7)$ & $15(1.2)$ & \\
\hline ASA class & & & 0.123 \\
\hline । & $6(23.1)$ & $520(42.2)$ & \\
\hline$\|$ & $18(69.2)$ & $680(55.2)$ & \\
\hline
\end{tabular}


Table 1 Association between potential risk factors and surgical site infection (SSI) in elective foot and ankle surgery (Continued)

\begin{tabular}{|c|c|c|c|}
\hline Variables & Number (\%) of infection & Number (\%) of non-infection & $p$ value \\
\hline III or greater & $2(7.7)$ & $33(2.7)$ & \\
\hline Intraoperative antibiotics & $16(61.5)$ & $791(64.2)$ & 0.779 \\
\hline Postoperative antibiotics & $17(65.4)$ & $724(58.7)$ & 0.494 \\
\hline Drainage use & $19(73.1)$ & $713(57.8)$ & 0.119 \\
\hline $\mathrm{TP}(<60 \mathrm{~g} / \mathrm{L})$ & $2(7.7)$ & $65(5.3)$ & 0.229 \\
\hline ALB (<35 g/L) & $7(26.9)$ & $18(1.5)$ & $<0.001$ \\
\hline GLOB (> 40 g/L) & $4(15.4)$ & $43(3.5)$ & 0.002 \\
\hline $\mathrm{A} / \mathrm{G}(<1.2)$ & $7(26.9)$ & $64(5.2)$ & $<0.001$ \\
\hline ALT $(>40 \mathrm{U} / \mathrm{L})$ & $4(15.6)$ & $62(5.0)$ & 0.019 \\
\hline AST (> 35 U/L) & $4(15.4)$ & $47(3.8)$ & 0.011 \\
\hline ALP (> upper limit) & $2(7.7)$ & $17(1.4)$ & 0.020 \\
\hline FBG (mmol/L) & $5.9 \pm 2.8$ & $5.3 \pm 1.4$ & 0.042 \\
\hline WBC $\left(>10 \times 10^{9} / L\right)$ & $3(11.5)$ & $44(3.6)$ & $<0.001$ \\
\hline NEUT $\left(>6.3 \times 10^{9} /\right.$ L) & $5(19.2)$ & $59(4.8)$ & $<0.001$ \\
\hline $\operatorname{LYM}\left(>3.2 \times 10^{9} / \mathrm{L}\right)$ & $3(11.5)$ & $86(7.0)$ & 0.468 \\
\hline RBC (< lower limit) & $12(46.2)$ & $14(53.8)$ & $<0.001$ \\
\hline HGB (< lower limit) & $16(61.5)$ & $10(38.5)$ & $<0.001$ \\
\hline HCT (< lower limit) & $4(15.4)$ & $97(7.9)$ & 0.163 \\
\hline MCHC (< lower limit) & $4(15.4)$ & 22 (84.6) & $<0.001$ \\
\hline PLT (< lower limit) & $2(7.7)$ & $24(92.3)$ & $<0.001$ \\
\hline PDW (< lower limit) & $3(11.5)$ & $23(88.5)$ & 0.034 \\
\hline
\end{tabular}

$R B C$ red blood cell, reference range: females $3.5-5.0 / 10^{12} / \mathrm{L}$ and males $4.0-5.5 / 10^{12} / \mathrm{L} ; H G B$ hemoglobin, reference range: females $110-150 \mathrm{~g} / \mathrm{L}$ and males $120-160$ $\mathrm{g} / \mathrm{L} ; A L B$ albumin; $F B G$ fasting blood glucose; $A L P$ alkaline phosphatase, 50-135 u/L; $H C T$ hematocrit, $40-50 \%$; $M C H C$, mean corpuscular hemoglobin concentration, 316-354 g/L; WBC white blood cell; NEUT neutrophil; LYM lymphocyte; PLT platelet, 100-300 × 109/L; TP total protein; ALB albumin; GLOB globulin; PDW platelet distribution width, reference $12-18.1 \%$

factors were identified to be associated with SSI. Delay of each day before operation was associated with $21 \%$ increased risk of SSI (OR, 1.21; 95\% CI, 1.09 to1.30). With reference to normal FBG level $(<6.1 \mathrm{mmol} / \mathrm{L})$, the risk of SSI was increased by $17 \%$ with each increment of 1 $\mathrm{mmol} / \mathrm{L}$ (OR, 1.17; 95\% CI, 1.04 to1.28). Compared to normal ALB level $(\geq 35 \mathrm{~g} / \mathrm{L})$ or NEUT count $(1.8-6.3 \times$ $\left.10^{9} / \mathrm{L}\right)$, the abnormal level was associated with 2.33 times and 1.72 times increased risk of SSI, respectively. The risk of SSI in patients with implantation of allograft or bone substitute was 3.76 times as those with none (OR, 3.76; 95\% CI, 1.51-5.30). The results are detailed in Table 2. The Hosmer-Lemeshow test showed the adequate fitness of the final model $\left(\chi^{2}=4.469, p=0.527\right.$; Nagelkerke's $R^{2}=0.377$ ).

\section{Discussion}

In this study, we found the overall incidence rate of SSI following elective foot and ankle surgery was $2.1 \%(1.3 \%$ for superficial and $0.8 \%$ for deep SSI). Several independent risk factors associated with SSI were identified, including prolonged preoperative stay, implantation of allograft or bone substitute, elevated FBG level, lower
ALB level $(<35 \mathrm{~g} / \mathrm{L})$, and increased NEUT count. Patients with SSI had 2.5 times prolonged total hospital stay as those with non-SSI (26.2 vs 10.3 days).

The incidence rate of SSI in foot and ankle surgery was reported to be varied, from 1.4 to $13.2 \%[4,8,9]$, largely due to the differences in definitions of SSI, study design, medical conditions, interventions, or follow-up period. Wiewiorski et al. [11] conducted a prospective study of 290 elective foot and ankle procedures and found the overall prevalence of wound complication of $16.9 \%$, with $1.4 \%$ for deep infections requiring irrigation and debridement. Zgonis et al. [8] evaluated the efficacy

Table 2 Multivariate analyses of risk factors associated with SSI in elective foot and ankle surgery

\begin{tabular}{lll}
\hline Variable & OR $(\mathbf{9 5 \%} \mathrm{Cl})$ & $\boldsymbol{p}$ value \\
\hline Preoperative stay (increase of each day) & $1.21(1.09$ to 1.30$)$ & 0.002 \\
Allograft or bone substitute & $3.76(1.51$ to 5.30$)$ & $<0.001$ \\
ALB level $<35 \mathrm{~g} / \mathrm{L}$ & $2.33(1.19$ to 3.05$)$ & 0.021 \\
NEUT $>6.3 \times 10^{9} / \mathrm{L}$ & $1.72(1.27$ to 2.12$)$ & 0.039 \\
FBG level (increase in each unit) & $1.17(1.04$ to 1.26$)$ & $<0.001$ \\
\hline
\end{tabular}

$A L B$ albumin, NEUT neutrophil, $O R$ odds ratio, $C I$ confidence interval 
of prophylactic use of antibiotics in elective foot and ankle surgery, but did not find the significant difference of SSI rate between patients who received preoperative antibiotic use and those did not (1.6\% vs $1.4 \%)$. In another study focusing on DM patients, SSI rate of $3.5 \%$ was observed, being $9.5 \%$ in DM patients and $2.4 \%$ in non-DM patients $(p$ $<0.001$ ) [16]. Despite with different settings, these studies provided more data to counsel patients about risk of SSI, facilitating the operative decision-making and perioperative optimal interventions.

Compared to the elective knee and hip arthroplasty, the incidence rate of SSI following elective foot and ankle surgery was higher, approximately 5 to 10 times high as that $[3,17]$. Ralte et al. [9] attributed this to the abundance of eccrine sweat glands and the hot and humid environment that caused microbiological flora in the feet. Additionally, the anatomical relationship of the toes is also an important contributor, which might weaken the efficacy of the preoperative skin disinfection. Tachibana [18] found the forefoot was more densely populated with microorganisms than other areas of the foot. Furthermore, it had been demonstrated that eliminating bacteria from the forefoot prior to surgery was more difficult $[19,20]$. In this study, we found a trend towards a higher incidence of SSI following forefoot surgeries $(2.5 \%$ vs $1.0 \%)$, although the result was nonsignificant, which, we thought, was primarily decided by the limited number of SSI cases $(n=26)$. Despite this, the future attention should focus on optimization of local surgical conditions and formation of a more reasonable antibiotic prophylaxis program aimed at the forefoot.

In the present study, higher FBG level rather than diagnosis of previous DM was identified as an independent risk factor for postoperative SSI, and increase of 1 $\mathrm{mmol} / \mathrm{L}$ in $\mathrm{FBG}$ was associated with $17 \%$ increased risk of SSI. This seemingly contradictory result did reflect the importance of blood glucose control for the prevention of SSI in patients with DM. Indeed, the role of history of DM in SSI has always been in controversy. In the study by Wiewiorski et al. [11], authors did not observe the independent association of SSI with DM in elective foot and ankle surgeries, which should be treated in the context of inadequate samples $(n=290)$. In a prospective cohort of 1465 foot and ankle surgeries conducted by Wukich et al. [16], DM-related peripheral neuropathy rather than DM itself was identified to be strongly associated with the development of SSI. Similarly, in a study of 21,854 diabetic patients, the authors found the number of comorbid conditions associated with DM had more predictive value than either DM or blood glucose level for occurrence of SSI [21]. These findings suggested that strict glycemic control (or HbA1c) and identification of comorbid conditions associated with DM made more sense than DM diagnosed by medical history, and they could become a routine part of preoperative comorbidity management.

The relationship between hypoalbuminemia and postoperative complications has been well established in orthopedic surgery and other subspecialties of surgery [22, 23], but was firstly reported as far as we know. Generally, patients who underwent elective foot and ankle surgeries were younger than those undergoing hip or knee arthroplasty, and in a certain proportion of hospitals, albumin was not routinely tested. In this study, the average age of included patients was 45 years, and only $2 \%$ of them had hypoalbuminemia (25/ 1259 ) in the context of routine test of preoperative albumin. Therefore, in such a low proportion (2\%), it might be very difficult to obtain the significant result via multivariate analyses in studies with small sample or without readily available albumin data. By far, several studies have demonstrated the improved outcomes via optimization of nutritional status prior to major surgery, including abdominal surgery, arthroplasties, or orthopedic trauma [24-26]. However, serum albumin is more often used to be a prognostic or nutritional marker, because simply restoring albumin level might not improve clinical outcomes [27].

Allograft or bone substitute as a risk factor for SSI was also firstly reported in this study and had the strongest association (OR, 3.76). On the one hand, the increased tension of skins around operative site or operative cavity after implantation of allograft or bone substitute may cause effusion of tissue fluid [28]. On the other hand, the graft of tissues does not have blood flow [29], which should be also considered as a potential cause for infection. But due to the limited cases (17 cases), this association was not definitive and its mechanism requires to be further investigated. The role of preoperative stay in risk of SSI in elective foot and ankle surgery should be rationally treated because the causes might be multifactorial, such as more time needed for optimization of comorbidities, unavailability of operation resources, weekends, or holidays [30,31]. About $5 \%$ of patients in this study had an increased neutrophil count $(>6.3 \times$ $10^{9} / \mathrm{L}$ ), in whom the incidence rate of SSI was $7.8 \%$, significantly higher than those with normal range of neutrophil count. In other studies of elective arthroplasties or others, the similar results were found $[32,33]$. These 3 factors might not be modified or optimized pertinently, but they could provide more references for surgeons to evaluate the risk of SSI following elective foot and ankle surgeries.

The major strengths of this study included its large sample size and multiple factors included for adjustment. Some limitations should also be mentioned. Firstly, the retrospective design compromised the accuracy and reliability in data collection, because the data depended on the documentations in the EMR. Secondly, 
the incidence rate of SSI might be underestimated due to our imperfect follow-up strategy. It was likely that one patient who developed a slight SSI that was untreated to resolve might report no SSIs occurring. Thirdly, as with every multivariate logistic regression analysis, there remain residual confounding effects, because of the unmeasured or unanticipated variables. Fourth, we could not obtain the data on patients' surgical site care or compliance with medication and functional exercises.

In summary, the incidence rate of SSI following elective foot and ankle surgery was low, but relatively high for forefoot surgeries. Prolonged preoperative stay, allograft or bone substitute, elevated FBG level, ALB level, and NEUT count were identified to be independently associated with SSI. Although not modifiable, they do help in patient counseling, SSI risk assessment, and stratifying patients, and should be kept in mind through perioperative period.

\section{Abbreviations \\ SSI: Surgical site infection; BMI: Body mass index; EMR: Electronic medical records; ASA: American Society of Anesthesiologists; CDC: Center for Disease Control; DM: Diabetes mellitus; SD: Standard deviation; OR: Odds ratio; RBC: Red blood cell; HGB: Hemoglobin; ALB: Albumin; FBG: Fasting blood glucose; ALP: Alkaline phosphatase; HCT: Hematocrit; MCHC: Mean corpuscular hemoglobin concentration; WBC: White blood cell; NEUT: Neutrophil; LYM: Lymphocyte; PLT: Platelet; TP: Total protein; GLOB: Globulin; PDW: Platelet distribution width; MRSA: Methicillin-resistant Staphylococcus aureus; MSSA: Methicillin-susceptible Staphylococcus aureus; MRCNS: Methicillin-resistant coagulase-negative Staphylococci}

\section{Acknowledgements}

We are grateful to $L$ Lin and $K$ Zhang of the Department of Orthopedics, and to K Ling and T Zhang of the Department of Statistics and Applications for their kind assistance.

\section{Authors' contributions}

Haitao Zhao designed the study; Jinghong Meng, Shiji Qin, and Yansen Li inquired the EMR for data on variables of interest; Fengqi Zhang and Tao Sun searched the relevant literature and analyzed and interpreted the data; Jinghong Meng and Yanbin Zhu wrote the manuscript, and Haitao Zhao approved the final version of the manuscript. The authors read approved the final manuscript

\section{Funding}

No organization sponsored the research.

\section{Availability of data and materials}

All the data will be available upon motivated request to the corresponding author of the present paper.

\section{Ethics approval and consent to participate}

This study was approved by the ethics committee of the 3rd Hospital of Hebei Medical University. Informed consent was obtained from all the participants.

\section{Consent for publication}

Written informed consent was obtained from each patient to authorize the publication of their data.

\section{Competing interests}

The authors declare that they have no competing interests.

\section{Author details}

'Department of Rheumatology and Immunology, The 3rd Hospital of Hebei Medical University, Shijiazhuang 050051, Hebei, People's Republic of China. ${ }^{2}$ Department of Orthopaedic Trauma Center, The 3rd Hospital of Hebei Medical University, Shijiazhuang 050051, Hebei, People's Republic of China. ${ }^{3}$ Department of Foot and Ankle Surgery, The 3rd Hospital of Hebei Medical University, No. 139 Ziqiang Road, Shijiazhuang 050051, Hebei, People's Republic of China. ${ }^{4}$ Department of Bone Tumor, The 3rd Hospital of Hebei Medical University, Shijiazhuang 050051, Hebei, People's Republic of China.

Received: 3 December 2019 Accepted: 23 September 2020

Published online: 01 October 2020

\section{References}

1. Magill SS, et al. Multistate point-prevalence survey of health care-associated infections. N Engl J Med. 2014;370(13):1198-208.

2. Liang $Z$, et al. Surgical site infection following elective orthopaedic surgeries in geriatric patients: incidence and associated risk factors. Int Wound J. 2019;16(3):773-80.

3. Zhu Y, et al. Risk factors for periprosthetic joint infection after total joint arthroplasty: a systematic review and meta-analysis. J Hosp Infect. 2015; 89(2):82-9.

4. Wukich DK, et al. Postoperative infection rates in foot and ankle surgery: a comparison of patients with and without diabetes mellitus. J Bone Joint Surg Am. 2010;92(2):287-95.

5. Plowman $\mathrm{R}$, et al. The rate and cost of hospital-acquired infections occurring in patients admitted to selected specialties of a district general hospital in England and the national burden imposed. 47(3):209.

6. Coello R, et al. Adverse impact of surgical site infections in English hospitals. J Hosp Infect. 60(2):103.

7. Savage JW, Anderson PA. An update on modifiable factors to reduce the risk of surgical site infections. Spine J. 2013;13(9):1017-29.

8. Zgonis T, Jolly GP, Garbalosa JC. The efficacy of prophylactic intravenous antibiotics in elective foot and ankle surgery. J Foot Ankle Surg. 2004;43(2):97-103.

9. Ralte $\mathrm{P}$, et al. The effect of strict infection control policies on the rate of infection after elective foot and ankle surgery: a review of 1737 cases. Bone Joint J. 2015:97-b(4):516-9.

10. Mulligan RP, et al. Preoperative risk factors for complications in elective ankle and hindfoot reconstruction. Foot Ankle Spec. 2018;11(1):54-60.

11. Wiewiorski $M$, et al. Risk factors for wound complications in patients after elective orthopedic foot and ankle surgery. Foot Ankle Int. 2015;36(5):479-87.

12. Paul Butterworth AT. Alex Barwick, Robert Hermann. The use of prophylactic antibiotics in podiatric foot and ankle surgery. Infec Dis Health. 2017;22:6-11.

13. Organ S. Guideline for prevention of surgical site infection, 1999; 1999.

14. Chen $Y$, et al. Association of body mass index and age with incident diabetes in Chinese adults: a population-based cohort study. BMJ Open. 2018;8(9):e021768.

15. Zhu Y, et al. Incidence and risks for surgical site infection after adult tibial plateau fractures treated by ORIF: a prospective multicentre study: SSI after tibial plateau fracture. Int Wound J. 2017;14(6).

16. Wukich DK, et al. Surgical site infections after foot and ankle surgery: a comparison of patients with and without diabetes. Diabetes Care. 2011; 34(10):2211-3

17. Wukich DK. et al, Postoperative infection rates in foot and ankle surgery: a comparison of patients with and without diabetes mellitus. 2010;8(2):146-7.

18. Tachibana DK. Microbiology of the foot. Annu Rev Microbiol. 1976;30:351-75.

19. Ostrander RV, Botte MJ, Brage ME. Efficacy of surgical preparation solutions in foot and ankle surgery. J Bone Joint Surg Am. 2005;87(5):980-5.

20. de Bengoa Vallejo RB, et al. Preoperative skin and nail preparation of the foot: comparison of the efficacy of 4 different methods in reducing bacterial load. J Am Acad Dermatol. 2009;61(6):986-92.

21. Domek N, et al. Association between hemoglobin a1c and surgical morbidity in elective foot and ankle surgery. J Foot Ankle Surg. 2016;55(5):939-43.

22. Yuwen $P$, et al. Albumin and surgical site infection risk in orthopaedics: a meta-analysis. BMC Surg. 17(1):7.

23. Hennessey DB. et al, Hypoalbuminaemaia is an independent risk factor for the development of surgical site infection following gasgtrointestinal surgery. 2011;158(2):385-5.

24. Evans DC, et al. Nutrition optimization prior to surgery. Nutr Clin Pract. 29(1):10-21.

25. Jie B, et al. Impact of preoperative nutritional support on clinical outcome in abdominal surgical patients at nutritional risk. Nutrition. 28(10). 
26. Eneroth M, Olsson U-B, Thorngren K-GR. Nutritional supplementation decreases hip fracture-related complications. Clin Orthop Relat Res. 451(451):212-7.

27. Vanek and V. W. The use of serum albumin as a prognostic or nutritional marker and the pros and cons of IV albumin therapy. Nutr Clin Pract. 13(3): p. 110-122.

28. Liu JH, Z.T., Dai XW, Analysis of related factors of infection after large allograft bone and joint transplantation. Modern Nurs, 2004. 10(1): p. 220-222

29. B, L., Risk management of infective diseases of allograft. Orthopedic Journal of China, 2009. 17(15): p. 1164-1166.

30. Ma T, et al. Modifiable factors as current smoking, hypoalbumin, and elevated fasting blood glucose level increased the SSI risk following elderly hip fracture surgery. J Investig Surg. 2019.

31. Meng, D., et al., Patient and perioperative factors influencing the functional outcomes and mortality in elderly hip fractures.

32. Perry J. The assessment of neutrophil cd64 count as an early warning marker of joint replacement infection. Arch Orthop Trauma Surg. 133(10):1351-8.

33. Youn JK, et al. The effect of absolute neutrophil count (ANC) on early surgical site infection in implanted central venous catheter (ICVC). J Pediatr Surg. 2019; undefined(undefined): p. undefined.

\section{Publisher's Note}

Springer Nature remains neutral with regard to jurisdictional claims in published maps and institutional affiliations.

Ready to submit your research? Choose BMC and benefit from:

- fast, convenient online submission

- thorough peer review by experienced researchers in your field

- rapid publication on acceptance

- support for research data, including large and complex data types

- gold Open Access which fosters wider collaboration and increased citations

- maximum visibility for your research: over $100 \mathrm{M}$ website views per year

At $\mathrm{BMC}$, research is always in progress.

Learn more biomedcentral.com/submissions 